Review

\title{
Environmental, Economic, and Social Potentialities of Ornamental Vegetation Cultivated in Constructed Wetlands of Mexico
}

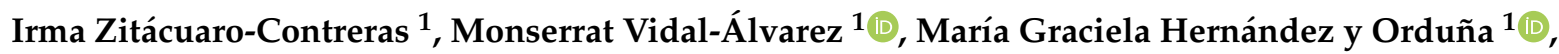 \\ Sergio Aurelio Zamora-Castro ${ }^{2}{ }^{-}$, Erick Arturo Betanzo-Torres ${ }^{3}{ }^{\circ}$, José Luis Marín-Muñiz ${ }^{1, *(1)}$ \\ and Luis Carlos Sandoval-Herazo ${ }^{3, * \mathbb{D}}$ \\ 1 El Colegio de Veracruz, Xalapa, Veracruz 91000, Mexico; izitacuaro@yahoo.com (I.Z.-C.); \\ monserrat.vidal@gmail.com (M.V.-Á.); gracielahernandez.orduna@gmail.com (M.G.H.y.O.) \\ 2 Facultad de Ingeniería, Construcción y Habitad, Universidad Veracruzana, Bv. Adolfo Ruíz Cortines 455, \\ Costa Verde, Boca del Rio, Veracruz 94294, Mexico; szamora@uv.mx \\ 3 Wetlands and Environmental Sustainabily Laboratory, División of Postgraduate Studies and Research, \\ Tecnológico Nacional de México/Instituto Tecnológico Superior de Misantla, Km 1.8 Carretera a Loma del Cojolite, \\ Veracruz 93821, Mexico; eabetanzot@itsm.edu.mx \\ * Correspondence: soydrew@hotmail.com (J.L.M.-M.); lcsandovalh@gmail.com (L.C.S.-H.)
}

Citation: Zitácuaro-Contreras, I.; Vidal-Álvarez, M.; Hernández y Orduña, M.G.; Zamora-Castro, S.A.; Betanzo-Torres, E.A.; Marín-Muñíz, J.L.; Sandoval-Herazo, L.C.

Environmental, Economic, and Social Potentialities of Ornamental Vegetation Cultivated in Constructed Wetlands of Mexico. Sustainability 2021, 13, 6267. https://doi.org/1 $0.3390 /$ su13116267

Academic Editor: Mustafa Al Aukidy

Received: 26 March 2021

Accepted: 26 May 2021

Published: 1 June 2021

Publisher's Note: MDPI stays neutral with regard to jurisdictional claims in published maps and institutional affiliations.

Copyright: (c) 2021 by the authors. Licensee MDPI, Basel, Switzerland. This article is an open access article distributed under the terms and conditions of the Creative Commons Attribution (CC BY) license (https:// creativecommons.org/licenses/by/ $4.0 /)$.

\begin{abstract}
Sustainable development implies the proper use of natural resources, such as water resources, but the environmental, economic, and social benefits should also be considered. In the water sector, for example, sanitation is one of the challenges that arise in rural localities. However, issues related to it may be solved with the use of constructed wetlands (CWs), which may allow the cultivation of ornamental plants with phytoremediation properties. Through a content analysis, the environmental, economic, and social potential of ornamental species used in Mexico for phytoremediation in CWs is documented in this study. The environmental potential of the plants was considered based on their phytoremediation properties. The economic potential was determined from research articles and from the National Agri-Food Information System. The social potential was analyzed considering the ornamental and artisanal use of the plants. In total, 21 species of phytoremediation plants were identified. Anthurium andreanum and Zantedeschia aethiopica stand out for their commercial value, reported in 2018 to be 272,875 and 30,318 USD, respectively, at the national level. The social potential was identified by the ornamental or artisanal use for flower arrangements and weaving. This study reveals that the use of CWs with ornamental vegetation is a sustainable option to clean wastewater, reuse water, and generate economic incentives. Thus, it is essential to provide the community with adequate training for the management of CWs in those sites that lack treatment systems.
\end{abstract}

Keywords: wastewater treatment; ornamental plants; sustainability

\section{Introduction}

Capitalist development is based on an extractivism system that, with a high demand for natural resources [1], causes great environmental deterioration. Therefore, at the international level, the 2030 Agenda for Sustainable Development includes 17 goals [2] that encompass the three aspects of sustainability: environmental, economic, and social. Making more efficient use of natural resources is an obligation.

In this regard, international policies provide guidelines for the desired development, such as the 2030 Agenda [2], a document that emphasized the commitment to protect the planet from environmental degradation through sustainable consumption, as well as the concept of sustainable development, which is based on meeting the needs of the present generation without compromising the ability of future generations to meet their own needs (Brundtland Report) [3]. 
However, it has been observed that the optimization of the use of natural resources has been put in standby at the international level [4]. The widespread development is the reason for a high consumption of natural resources. This may suggest that a reconversion of the production processes and the use and conservation of natural resources are crucial. Investing in these changes is justified to save resources and generate less waste and thus reduce pollution [5].

Water and vegetation are among the natural resources with the greatest impact on the development of localities. In terms of water, in Mexico in 2017, of the 653 existing aquifers, $23.5 \%$ presented availability problems, as the volume of extraction exceeded that of recharge to the aquifer. This caused salinity problems and overexploitation of aquifers. In addition, of the 731 basins in the country, 13.4\% registered resource problems due to low availability and contamination [6]. Another factor that limits the availability of water is pollution. This may be due to the scarce infrastructure for wastewater treatment. Regarding this, in Mexico, in the urban public sector, only $53.9 \%$ of the discharged $215.1 \mathrm{~m}^{3} / \mathrm{s}$ are treated, and in the industrial sector, $38.3 \%$ of the generated $218.4 \mathrm{~m}^{3} / \mathrm{s}$ at the national level are treated [6]. These figures do not consider wastewater discharges without the corresponding permits. Therefore, the contamination of water bodies is of great magnitude.

Vegetation is another important resource that has been deteriorated by human activity. Toledo [7] and Rzedowski [8] have made estimates of the phanerogamic species (flowering plants and seeds) in Mexico, finding the existence of 220 registered families, with 2410 genera and 22,000 species, which puts the country in the first places of biodiversity worldwide. Among these species are those with ornamental flowers that can have commercial use. More than 1000 species and varieties of ornamental plants have been identified in Mexico [9].

In addition to their commercial value, some species of ornamental plants can be used for phytoremediation or purification of polluted waters, in particular, of wastewater discharges of urban public origin or industrial processes. Therefore, they are of interest to this study, due to their use as macrophyte plants typical of natural wetlands, with physical and biological characteristics of absorption/adsorption of contaminants present in soil, air, or water $[10,11]$. These types of plants are useful in sanitation processes through CWs, which are the subject of academic research, considering their possible use in small towns [12-15]. CWs have been engineered as systems that operate with physical, chemical, and biological processes to remove most of the contaminants from water. The main CWs components are substrates, microorganisms, and vegetation [16,17].

CWs are an economical and ecologically viable option for cleaning wastewater. They consist of cells filled with a granular medium, which functions as a support for the planted vegetation. They allow the development of microorganisms that eliminate a large part of organic and inorganic pollutants through aerobic processes [18-20]. The vegetation planted in CWs as a phytoremediation agent can be of three types: (1) emergent, if the aerial biomass protrudes from the surface, (2) floating, if the biomass and roots develop in the water, and (3) submerged, when they root to the substrate deposited in the cells without leaving a water column $[19,21]$.

In general, CWs are considered an option for the development of small towns because they do not require energy resources, they do not require specialized labor, and their process does not require chemical agents [19,22].

It is observed that, given the problem of lack of wastewater sanitation in small towns, CWs vegetated with ornamental plants are a viable option that may be attractive from a social perspective, as it promotes flower production. However, this sanitation infrastructure is not popular in Mexico, largely due lack of knowledge about it and its components, in particular, vegetation [18]. This study aims at analyzing the environmental, economic, and social potential of ornamental species used in Mexico for phytoremediation in constructed wetlands.

This study can help select appropriate plant species to improve the removal of pollutants. 
On the other hand, it can improve the aesthetics of CWs, making them attractive to the eye with sophisticated and innovative forms, as observed for the Olentangy River Wetland Research Park in Ohio State University, Columbus, a wetland shaped like a kidney, allusive to the belief that wetlands are the kidneys of plants [23].

Likewise, CWs are recommended to be built in small towns where government investment is scarce. These sanitation systems require small investments for their construction, operation, and maintenance [24]. In social participation schemes, operation and maintenance can be carried out by the community.

\section{Materials and Methods}

The research was carried out through content analysis [19], predominantly on qualitative research. The literature review methodology in our study (see Figure 1) was based on Colicchia and Strozzi [25], Piñar [26], and Ponis and Ntalla [27].

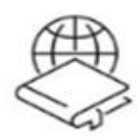

\section{Literature review methodology}

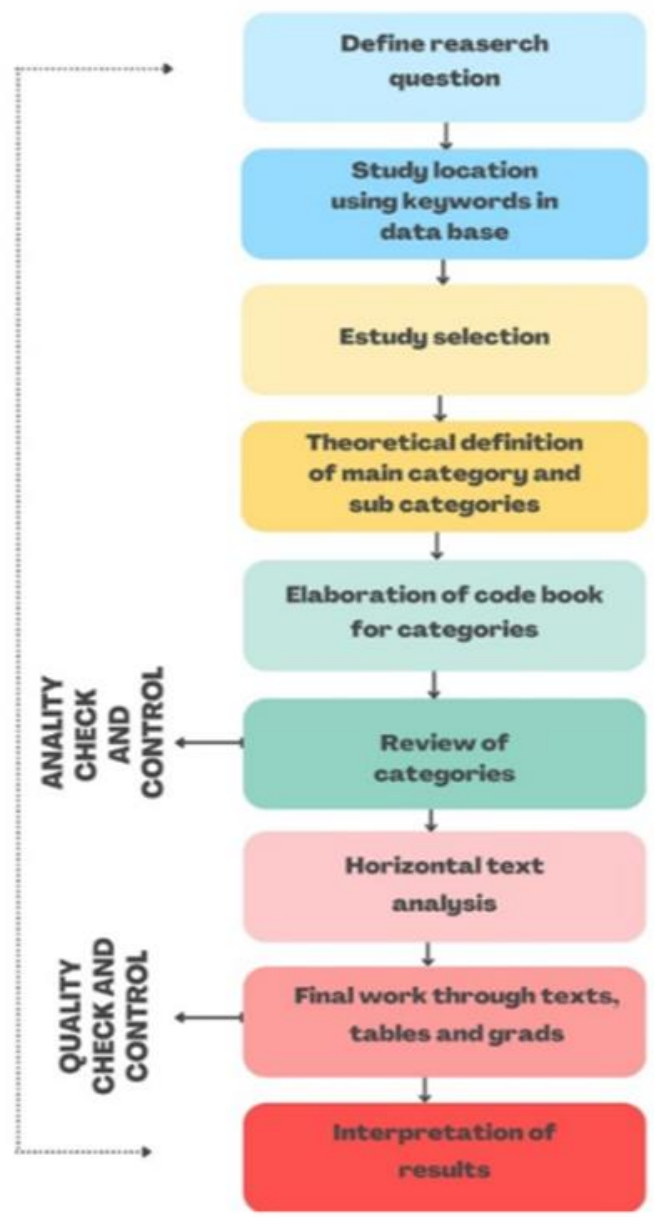

Figure 1. Literature review methodology.

The methodological steps of this study are shown in Figure 1. We started by defining the research question, then interpreted the results, following a total of nine stages with a quality control process in the final stages.

The research question we addressed was: "What is the environmental, economic, and social potential of ornamental species in Mexico for phytoremediation?"

We searched databases using researchgate.net, academia.edu, TESIUNAM; in addition, articles on the web of science, Scopus, and ISI Web of Knowledge were reviewed, as well as 
publications of Instituto Mexicano de Tecnología del Agua, a database that reports ornamental species used in Mexico and contains free-access resources. For all search engines, we used keywords such as phytoremediation, ornamental plants, constructed wetlands (CW), and pollutant removal. We focused on ornamental vegetation (unit of analysis) used for phytoremediation in CW, due to its high pollutant removal efficiency in water and its attractiveness for cultivation in $\mathrm{CW}$, to which it gives a pleasant appearance, facilitating their acceptance. We focused on the characterization, commercialization, and uses of ornamental plants with phytoremediation properties to describe a possible second use of these plants, besides the decorative one.

A horizontal text analysis [26] allowed us to identify common properties of contaminant and parameters that were at the basis of different studies on CWs. Furthermore, a quantitative technique was used to determine the frequency of the parameters analyzed and the number of species identified as ornamental with phytoremediation properties. Likewise, the plants were classified by their potential to purify the soil and air.

To detect the environmental value of the ornamental plants used in phytoremediation processes, we reviewed studies that evaluated the purification of wastewater by removing pollutants identified by the parameters of Biological Oxygen Demand $\left(\mathrm{BOD}_{5}\right)$, Chemical Oxygen Demand (COD), Fats and Oils (G and A), Total Suspended Solids (SST), Total Dissolved Solids (TDS), Fecal Coliforms (CF), Chloride (Cl), Phosphorus (P), Nitrogen $(\mathrm{N})$, Ammonium $\left(\mathrm{NH}_{4}\right)$, Phosphate $\left(\mathrm{PO}_{4}\right)$, Ibuprofen $\left(\mathrm{C}_{13} \mathrm{H}_{18} \mathrm{O}_{2}\right)$, Arsenic (As), Sulfate $\left(\mathrm{SO}_{4}\right)$, Volatile solids (SV), and Nitrate $\left(\mathrm{NO}_{3}\right)$. In this regard, from a total of 55 papers, 41 were selected because they contain reports of removal efficiencies greater than $50 \%$. The information was integrated into an excel database with variables such as species, type of parameter analyzed, removal efficiency, and type of wetland.

Regarding the economic value of the species used in wetlands, the market value of ornamental plants was recorded on the basis of publications and reports of the Sistema de Información Agroalimentaria de Consulta (SIACON-NG), with information as of 2018 reported by the Secretaría de Agricultura, Ganadería, Pesca y Alimentación (SAGARPA). Through a database generated with searches in the SIACON-NG on the selected species with environmental potential, information on the commercial value at the national level was integrated.

The analysis of the social potential (ornamental, artisanal, medicinal, and food) of the ornamental species cultivated in the selected constructed wetlands was carried out through 21 publications that addressed this aspect.

In total, 84 publications were analyzed to support the information we present, of which 22 scientific articles used for the review are included in the Journal citation report, (47\%); the additional bibliographic material was obtained from reliable primary and secondary sources.

\section{Results}

Environmental potential shown in Figure 2. from the analysis of CW projects where ornamental flowers were used as a phytoremediation process, 21 species of plants were identified (Table 1). The role of these species in the wastewater purification process consists in absorption/filtering of pollutants through the roots [12,16]. In the same way, they can degrade chemical compounds, including drugs such as Ibuprofen [13]. 


\section{Classification of the social values of ornamental plants used in constructed wetlands}

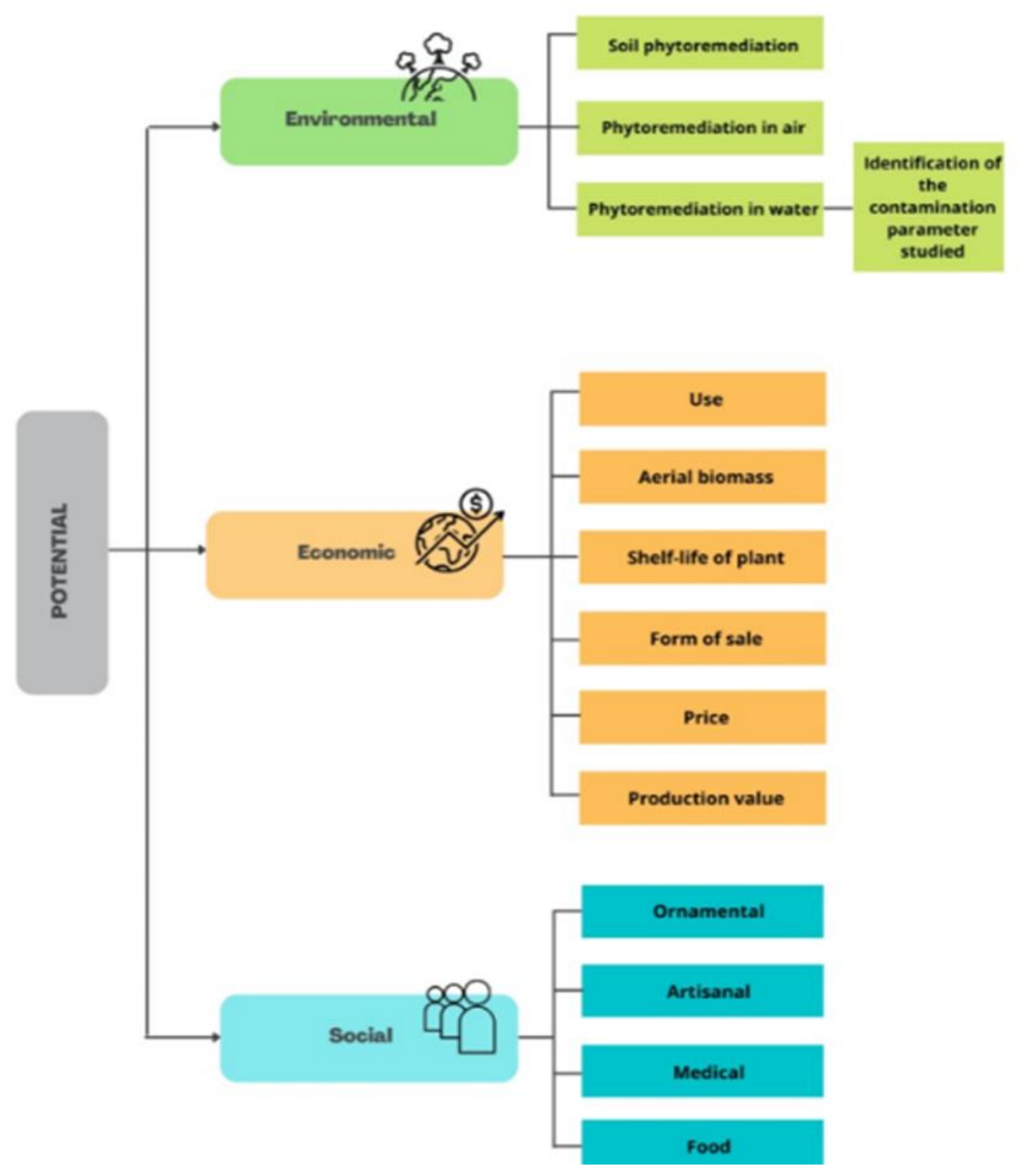

Figure 2. Environmental, economic, and social potentialities analyzed in this study.

\subsection{Environmental Potential}

The polluting parameters mostly analyzed in phytoremediation processes in constructed wetlands are shown in Figure 3. $\mathrm{BOD}_{5}$ and $\mathrm{COD}$ of urban public wastewater discharges were the most examined parameters. They both describe the degree of water contamination. The ornamental species most commonly used in phytoremediation appeared to be Zantedeschia aethiopica and Spathiphyllum blandum. 
Table 1. Vegetation for phytoremediation growing in constructed wetlands.

\begin{tabular}{|c|c|c|c|c|c|c|}
\hline \multirow[b]{2}{*}{ Scientific Name } & \multirow[b]{2}{*}{ Measured Pollutant } & \multirow[b]{2}{*}{$\begin{array}{l}\text { Environmental } \\
\text { Potential }\end{array}$} & \multicolumn{2}{|c|}{ Economic Potential } & \multirow{2}{*}{$\begin{array}{c}\text { Social Potential } \\
\text { (Ornamental, } \\
\text { Artisanal, } \\
\text { Medicinal, Food) * }\end{array}$} & \multirow[b]{2}{*}{ Reference } \\
\hline & & & $\begin{array}{c}\text { Use/Aerial } \\
\text { Biomass/Shelf-Life } \\
\text { of Plant }\end{array}$ & $\begin{array}{l}\text { Form of } \\
\text { Sale/Price }\end{array}$ & & \\
\hline Pragmites australis & $\begin{array}{l}\mathrm{BOD}_{5}, \mathrm{G} \text { and } \mathrm{A} \\
\quad \mathrm{N}, \mathrm{PO}_{4}\end{array}$ & $\begin{array}{l}\text { Phytoremediation } \\
\text { (water) }\end{array}$ & $\begin{array}{l}\text { Artisanal/pot } \\
\text { plant/leaf } \\
\text { and flower }\end{array}$ & $\begin{array}{l}\text { Local trade } \\
\text { in USD }\end{array}$ & Artisanal, medicinal & Shaltout, et al. [27] \\
\hline Gladiolus spp & $\begin{array}{l}\mathrm{BOD}_{5}, \mathrm{G} \text { and } \mathrm{A} \\
\quad \mathrm{N}, \mathrm{PO}_{4}\end{array}$ & $\begin{array}{l}\text { Phytoremediation } \\
\text { (water) }\end{array}$ & $\begin{array}{l}\text { Ornamental/flower } \\
\text { arrangement, } \\
\text { pot/flower }\end{array}$ & $\begin{array}{l}\text { Local trade }(80 \% \\
\text { in Mexico/14.14 } \\
\text { per flower }\end{array}$ & $\begin{array}{l}\text { Ornamental, } \\
\text { medicinal }\end{array}$ & $\begin{array}{l}\text { Castañeda y Flores [28] } \\
\text { Manuja [29] } \\
\text { Ramos-García et al. [30] }\end{array}$ \\
\hline $\begin{array}{l}\text { Canna (indica, } \\
\text { hybrids) }\end{array}$ & $\begin{array}{c}\mathrm{BOD}_{5}, \mathrm{COD}, \mathrm{TSS}, \mathrm{Cl} \\
\text { P, N, SV }\end{array}$ & $\begin{array}{l}\text { Phytoremediation } \\
\text { (water) }\end{array}$ & $\begin{array}{l}\text { Ornamental/ } \\
\text { pot/plant }\end{array}$ & Local trade & $\begin{array}{l}\text { Ornamental, food, } \\
\text { medicinal }\end{array}$ & $\begin{array}{c}\text { Sandoval et al. [31] } \\
\text { Sandoval-Herazo et al. [32] } \\
\text { Zurita \& White [33] } \\
\text { Zurita et al. [12] } \\
\text { Al-Snafi [34] } \\
\text { Amaya [35] } \\
\text { Gallegos [36] } \\
\text { Martínez [37] } \\
\text { CONABIO [38] } \\
\end{array}$ \\
\hline Iris (japonica) & $\begin{array}{c}\mathrm{BOD}_{5}, \mathrm{COD}, \mathrm{G} \text { and } \\
\mathrm{A}, \mathrm{CF}, \mathrm{NH}_{4}, \mathrm{PO}_{4}\end{array}$ & $\begin{array}{l}\text { Phytoremediation } \\
\text { (water) }\end{array}$ & $\begin{array}{l}\text { Ornamental/ } \\
\text { pot/plant }\end{array}$ & Local trade & Ornamental & $\begin{array}{c}\text { Sandoval et al. [31] } \\
\text { Garzón et al. [23] } \\
\text { Tejeda et al. [39] }\end{array}$ \\
\hline $\begin{array}{l}\text { Heliconia (sp, stricta, } \\
\text { psittacorum, rostrata) }\end{array}$ & $\begin{array}{l}\mathrm{BOD}_{5}, \mathrm{COD}, \mathrm{G} \text { and } \\
\mathrm{A}, \mathrm{CF}, \mathrm{NH}_{4}, \mathrm{PO}_{4} \text { and } \\
\text { adaptation to } \\
\text { substrate }\end{array}$ & $\begin{array}{l}\text { Phytoremediation } \\
\text { (water) }\end{array}$ & $\begin{array}{l}\text { Ornamental / flower } \\
\text { arrangement / plant, } \\
\text { flower/7-25 days }\end{array}$ & $\begin{array}{c}\text { Local trade/ } 0.91 \\
\text { and } 1.26 \text { by } \\
\text { flower, } 10.11 \text { per } \\
\text { plant }\end{array}$ & Ornamental & $\begin{array}{l}\text { Sandoval et al. [31] } \\
\text { Garzón et al. [23] } \\
\text { Jerez [40] } \\
\text { Sosa [41] } \\
\text { Carrera [42] }\end{array}$ \\
\hline $\begin{array}{l}\text { Zantedeschia } \\
\text { aethiopica }\end{array}$ & $\begin{array}{l}\mathrm{BOD}_{5}, \mathrm{COD}, \mathrm{G} \text { and } \\
\mathrm{A}, \mathrm{SST}, \mathrm{CF}, \mathrm{Cl}, \mathrm{N}, \\
\mathrm{PO}_{4}, \mathrm{As}, \mathrm{SV}, \mathrm{NO}_{3}\end{array}$ & $\begin{array}{l}\text { Phytoremediation } \\
\text { (water) }\end{array}$ & $\begin{array}{l}\text { Ornamental/flower } \\
\text { arrangement, } \\
\text { pot/plant, } \\
\text { flower/8-10 days }\end{array}$ & $\begin{array}{l}\text { Local trade } / 4.04 \\
\text { per plant }\end{array}$ & Ornamental & $\begin{array}{c}\text { Sandoval-Herazo et al. [32] } \\
\text { Belmont et al. [43] } \\
\text { Belmont y Metcalfe [44] } \\
\text { Ramírez-Carrillo et al. [45] } \\
\text { Zurita et al. [12] } \\
\text { Zurita et al. [46] } \\
\text { Zurita \& White [33] } \\
\text { Zurita \& Carreón-Álvarez [47] } \\
\text { Garzón, et al. [23] } \\
\text { Hernández [48] } \\
\text { Tejeda et al. [39] } \\
\text { Marín-Muñiz et al. [19] } \\
\text { Sandoval-Herazo et al. [20] } \\
\text { Dennis et al. [49] } \\
\text { Pedraza [13] } \\
\text { Pedraza [50] } \\
\text { Martínez [37] } \\
\text { Pato et al. [51] }\end{array}$ \\
\hline Strelitzia reginae & $\begin{array}{l}\mathrm{BOD}_{5}, \mathrm{COD}, \mathrm{G} \text { and } \\
\mathrm{A}, \mathrm{CF}, \mathrm{NH}_{4}, \mathrm{PO}_{4}\end{array}$ & $\begin{array}{l}\text { Phytoremediation } \\
\text { (water) }\end{array}$ & $\begin{array}{l}\text { Ornamental/flower } \\
\text { arrangement, } \\
\text { pot/plant, } \\
\text { flower/18.8 days }\end{array}$ & $\begin{array}{c}\text { National } \\
\text { trade/30,318.34 } \\
\text { to } 2018,17.69 \\
\text { per plant }\end{array}$ & $\begin{array}{l}\text { Ornamental, } \\
\text { medicinal }\end{array}$ & $\begin{array}{c}\text { Zurita et al. [12] } \\
\text { Zurita et al. [52] } \\
\text { Zurita \& Carreón-Álvarez [47] } \\
\text { Merino-Solís et al. [53] } \\
\text { Garzón, et al. [23] } \\
\text { SIACON- NG [54] } \\
\text { Sánchez [55] } \\
\text { González [56] } \\
\text { Ramírez-Guerrero et al. [57] }\end{array}$ \\
\hline $\begin{array}{l}\text { Anthurium } \\
\text { andreanum }\end{array}$ & $\begin{array}{c}\mathrm{BOD}_{5}, \mathrm{COD}, \mathrm{CF}, \mathrm{P}, \\
\mathrm{N}, \mathrm{NH}_{4}, \mathrm{NO}_{3}\end{array}$ & $\begin{array}{l}\text { Phytoremediation } \\
\text { (water and air) }\end{array}$ & $\begin{array}{l}\text { Ornamental/flower } \\
\text { arrangement, } \\
\text { pot/plant, } \\
\text { flower/15-25 days }\end{array}$ & $\begin{array}{c}\text { National } \\
\text { trade } / 277,918.14 \\
\text { to } 2018,15.16 \\
\text { per plant }\end{array}$ & Ornamental & $\begin{array}{c}\text { Zurita et al. [12] } \\
\text { Zurita et al. [31] } \\
\text { Hernández [48] } \\
\text { Pato et al. [58] } \\
\text { Gayosso-Rodríguez et al. [59] } \\
\text { Pedraza [50] } \\
\text { SIACON- NG [54] }\end{array}$ \\
\hline Anemopsis californica & DTS, $\mathrm{Cl}, \mathrm{P}, \mathrm{As}, \mathrm{SO}_{4}$ & $\begin{array}{l}\text { Phytoremediation } \\
\text { (water) }\end{array}$ & Ornamental/pot/plant & Local trade & $\begin{array}{l}\text { Ornamental, } \\
\text { medicinal, food }\end{array}$ & Zurita et al. [46] \\
\hline Alpinia purpurata & $\begin{array}{l}\mathrm{BOD}_{5}, \mathrm{COD}, \mathrm{Cl}, \mathrm{P}, \\
\mathrm{N}, \mathrm{SV}\end{array}$ & $\begin{array}{l}\text { Phytoremediation } \\
\text { (water) }\end{array}$ & $\begin{array}{l}\text { Ornamental/flower } \\
\text { arrangement, } \\
\text { pot/plant, flower }\end{array}$ & Local trade & $\begin{array}{l}\text { Ornamental, } \\
\text { medicinal }\end{array}$ & $\begin{array}{c}\text { Méndez-Mendoza et al. [60] } \\
\text { Marín-Muñiz et al. [18] } \\
\text { Amaya [35] } \\
\text { Gallegos [36] } \\
\text { Sánchez [55] }\end{array}$ \\
\hline $\begin{array}{l}\text { Spathiphyllumwallisii/ } \\
\text { blandum }\end{array}$ & $\begin{array}{c}\mathrm{BOD}_{5}, \mathrm{COD}, \mathrm{G} \text { and } \\
\mathrm{A}, \mathrm{TSS}, \mathrm{CF}, \mathrm{P}, \mathrm{N}, \mathrm{PO}_{4}, \\
\mathrm{NH}_{4}, \mathrm{NO}_{3}, \\
\mathrm{C}_{13} \mathrm{H}_{18} \mathrm{O}_{2}\end{array}$ & $\begin{array}{l}\text { Phytoremediation } \\
\text { (water and air) }\end{array}$ & $\begin{array}{c}\text { Ornamental/flower } \\
\text { arrangement, } \\
\text { pot/planta/plant, } \\
\text { flower }\end{array}$ & $\begin{array}{l}\text { Local trade } / 4.04 \\
\text { per plant }\end{array}$ & $\begin{array}{l}\text { Ornamental, } \\
\text { medicinal }\end{array}$ & $\begin{array}{c}\text { Garzón, et al. [23] } \\
\text { Sandoval-Herazo et al. [20] } \\
\text { Pereira [61] } \\
\text { Martínez [62] } \\
\text { Plantas y Flores [63] } \\
\text { Sandoval et al. [64] }\end{array}$ \\
\hline $\begin{array}{l}\text { Hedychium } \\
\text { coronarium }\end{array}$ & $\begin{array}{l}\mathrm{BOD}_{5}, \mathrm{COD}, \mathrm{G} \\
\text { and } \mathrm{A}, \mathrm{CF}, \mathrm{Cl} \\
\mathrm{NH}_{4}, \mathrm{PO}_{4}, \mathrm{SV}\end{array}$ & $\begin{array}{l}\text { Phytoremediation } \\
\text { (water) }\end{array}$ & $\begin{array}{l}\text { Ornamental/pot/ } \\
\text { plant/plant }\end{array}$ & Local trade & $\begin{array}{l}\text { Ornamental, } \\
\text { medicinal, food }\end{array}$ & $\begin{array}{c}\text { Garzón et al. [23] } \\
\text { Hernández [48] } \\
\text { Pedraza [13] } \\
\text { Pedraza [50] } \\
\text { Amaya [35] } \\
\text { Gallegos [36] }\end{array}$ \\
\hline Alocasia $s p$ & $\begin{array}{l}\mathrm{BOD}_{5}, \mathrm{COD}, \mathrm{G} \text { and } \\
\mathrm{A}, \mathrm{TSS}, \mathrm{CF}, \mathrm{P}^{\prime} \mathrm{N}, \\
\mathrm{NH}_{4}, \mathrm{PO}_{4}, \mathrm{NO}_{3}\end{array}$ & $\begin{array}{l}\text { Phytoremediation } \\
\text { (water) }\end{array}$ & $\begin{array}{l}\text { Ornamental/pot/ } \\
\text { plant/plant }\end{array}$ & $\begin{array}{l}\text { Local trade/14.15 } \\
\text { per plant }\end{array}$ & $\begin{array}{l}\text { Ornamental, } \\
\text { medicinal }\end{array}$ & $\begin{array}{l}\text { Garzón et al. [23] } \\
\text { Romero [65] }\end{array}$ \\
\hline
\end{tabular}


Table 1. Cont.

\begin{tabular}{|c|c|c|c|c|c|c|}
\hline \multirow[b]{2}{*}{ Scientific Name } & \multirow[b]{2}{*}{ Measured Pollutant } & \multirow[b]{2}{*}{$\begin{array}{l}\text { Environmental } \\
\text { Potential }\end{array}$} & \multicolumn{2}{|c|}{ Economic Potential } & \multirow{2}{*}{$\begin{array}{c}\text { Social Potential } \\
\text { (Ornamental, } \\
\text { Artisanal, Medicinal, } \\
\text { Food) }{ }^{*}\end{array}$} & \multirow[b]{2}{*}{ Reference } \\
\hline & & & $\begin{array}{c}\text { Use/Aerial } \\
\text { Biomass/Shelf-Life } \\
\text { of Plant }\end{array}$ & $\begin{array}{l}\text { Form of } \\
\text { Sale/Price }\end{array}$ & & \\
\hline Lilium $s p$ & COD, TSS, $\mathrm{P}, \mathrm{NO}_{3}$ & $\begin{array}{l}\text { Phytoremediation } \\
\text { (water) }\end{array}$ & $\begin{array}{l}\text { Ornamental/flower } \\
\text { arrangement, } \\
\text { pot/plant, flower }\end{array}$ & $\begin{array}{l}\text { Local trade } / 13.64 \\
\text { per plant }\end{array}$ & $\begin{array}{l}\text { Ornamental, } \\
\text { medicinal }\end{array}$ & $\begin{array}{c}\text { Hernández [48] } \\
\text { Pedraza [13] } \\
\text { Pedraza [40] } \\
\text { Gardenia Creating Gardens } \\
\text { [66] }\end{array}$ \\
\hline Typha latifolia & $\begin{array}{c}\mathrm{BOD}_{5}, \mathrm{PO}_{4}, \mathrm{~N}, \mathrm{G} \text { and } \\
\mathrm{A}, \mathrm{NO}_{3}\end{array}$ & $\begin{array}{l}\text { Phytoremediation } \\
\text { (water) }\end{array}$ & $\begin{array}{l}\text { Artisanal/furniture/ } \\
\text { plant, leaves }\end{array}$ & Local trade & Artisanal, medicinal & $\begin{array}{c}\text { Bela y Chifa [67] } \\
\text { Hernández [48] } \\
\text { Marín-Muniz [17] } \\
\text { Grace et al. [68] } \\
\text { Pedraza [13] } \\
\text { Pedraza [50] } \\
\text { Hernández [48] }\end{array}$ \\
\hline Pontederia sagittata & $\mathrm{BOD}_{5}, \mathrm{COD}, \mathrm{NO}_{3}$ & $\begin{array}{l}\text { Phytoremediation } \\
\text { (water) }\end{array}$ & $\begin{array}{l}\text { Ornamental/flower } \\
\text { arrangement/plant, } \\
\text { flower, leaves }\end{array}$ & $\begin{array}{l}\text { Local trade } / 4.04 \\
\text { per plant }\end{array}$ & Ornamental & Olguín et al. [69] \\
\hline Hemerocallis & $\mathrm{BOD}_{5}, \mathrm{COD}, \mathrm{P}, \mathrm{NO}_{3}$ & $\begin{array}{l}\text { Phytoremediation } \\
\text { (water) }\end{array}$ & $\begin{array}{l}\text { Ornamental/flower } \\
\text { arrangement/plant, } \\
\text { flower/1 day }\end{array}$ & $\begin{array}{l}\text { Local trade } / 6.32 \\
\text { per plant }\end{array}$ & Ornamental & Zurita et al. [12] \\
\hline Cyperus papyrus & $\begin{array}{l}\mathrm{BOD}_{5}, \mathrm{COD}, \mathrm{G} \text { and } \\
\mathrm{A}, \mathrm{CF}, \mathrm{NH}_{4}, \mathrm{PO}_{4}\end{array}$ & $\begin{array}{l}\text { Phytoremediation } \\
\text { (water) }\end{array}$ & $\begin{array}{l}\text { Ornamental and } \\
\text { artisanal/pot/plant }\end{array}$ & $\begin{array}{l}\text { Local trade } / 3.54 \\
\text { per plant }\end{array}$ & $\begin{array}{l}\text { Ornamental, } \\
\text { artisanal }\end{array}$ & $\begin{array}{c}\text { Garzón et al. [23] } \\
\text { Programa Master Gardener [70] } \\
\text { Pedraza [13] } \\
\text { Pedraza [40] } \\
\text { Hernández [48] }\end{array}$ \\
\hline Dieffenbachia seguine & $\begin{array}{c}\mathrm{BOD}_{5}, \mathrm{~N}_{4} \mathrm{NH}_{4}, \\
\mathrm{PO}_{4}, \mathrm{NO}_{3}\end{array}$ & $\begin{array}{l}\text { Phytoremediation } \\
\text { (water) }\end{array}$ & $\begin{array}{l}\text { Ornamental/pot/ } \\
\text { plant, flower }\end{array}$ & $\begin{array}{l}\text { Local trade/17.69 } \\
\text { per plant }\end{array}$ & $\begin{array}{c}\text { Ornamental, } \\
\text { medicinal }\end{array}$ & $\begin{array}{c}\text { Martínez [62] } \\
\text { Missouri Botanical Garden [71] }\end{array}$ \\
\hline Zingiber spectabile & $\mathrm{COD}, \mathrm{NO}_{3}, \mathrm{PO}_{4}$ & $\begin{array}{l}\text { Phytoremediation } \\
\text { (water) }\end{array}$ & $\begin{array}{l}\text { Ornamental/flower } \\
\text { arrangement/plant, } \\
\text { flower, leaf/ } \\
\text { 8-14 days }\end{array}$ & $\begin{array}{c}\text { Local and } \\
\text { international } \\
\text { trade } / 0.71-1.16 \\
\text { per flower, } \\
\$ 12.63 \text { per plant }\end{array}$ & $\begin{array}{l}\text { Ornamental, } \\
\text { medicinal }\end{array}$ & $\begin{array}{l}\text { Cuevas [72] } \\
\text { Baltazar-Bernal et al. [73] }\end{array}$ \\
\hline Etlingera elatior & $\begin{array}{l}\text { Only was evaluated } \\
\text { the use of substrate }\end{array}$ & $\begin{array}{l}\text { Phytoremediation } \\
\text { (water) }\end{array}$ & $\begin{array}{l}\text { Ornamental/flower } \\
\text { arrangement/plant, } \\
\text { flower, leaf }\end{array}$ & $\begin{array}{l}\text { Local trade } / \$ 12.63 \\
\text { per plant }\end{array}$ & Ornamental & $\begin{array}{l}\text { Cuevas [72] } \\
\text { Carrera [42] }\end{array}$ \\
\hline
\end{tabular}

Ornamental (Ornamental: social festivities such as birthdays, religious holidays, Day of the Dead, decoration of streets and houses, flower arrangements, potted plant); ART (artisan; manufacture of furniture, baskets, necklaces, rattles, cellulose, fences, ceilings); Medicinal (medicinal); Food (food: sheet for, tamales, flour, stems, roots). * See description of Figure 2.

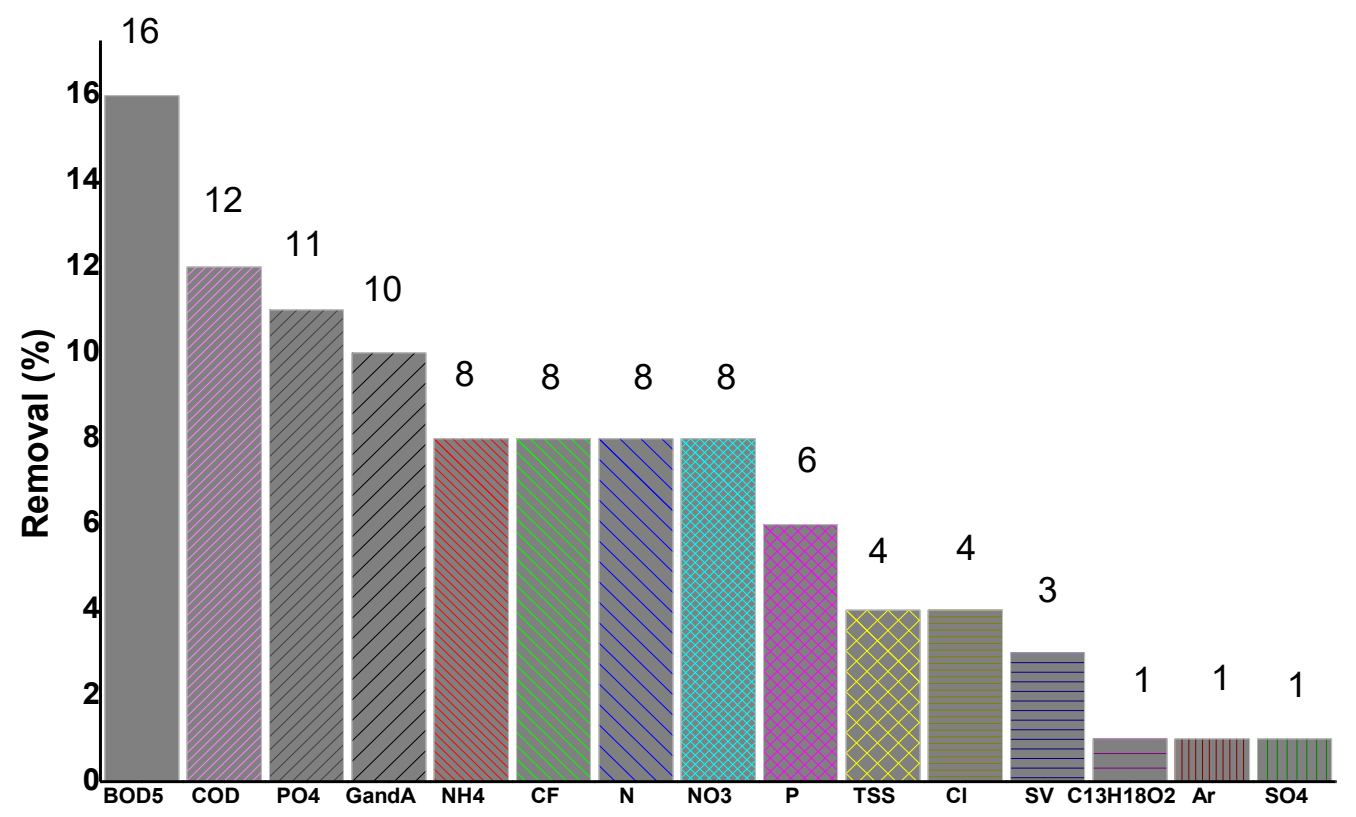

Contamination Parameter

Figure 3. Frequency of study of the contamination parameters analyzed in phytoremediation processes.

$\mathrm{BOD}_{5}$ reduction by plants oscillated between 70 and $89 \%$; likewise, COD reduction resulted to be $70-86 \%$, and reduction of $\mathrm{PO}_{4}$ and fats and oils were found to amount to $70-84 \%$ and $70-82 \%$, respectively. The choice of plants, in addition to their phytoremedi- 
ation properties, responded to several factors such as the type of wastewater discharge (municipal, industrial, others) and the climate.

\subsection{Economic Potential}

This may vary with respect to the intended use for the aerial mass of the plants and refers to the commercialization of cut flowers, foliage, stems, and plants in pots [9,74]. Other factors that may influence the commercialization are the regional and traditional uses of plants that vary locally, that is, customs and traditions influence the demand of certain specific species in different regions and seasons of the year.

As a consequence of the recognition of the ornamental value of plants, in Mexico the production of these species has increased. The economic value of these plants is registered by the SAGARPA, through the Information System of Agrifood information. The main contributions by plant species are shown in Table 2, which also includes Anthurium and Zantedeschia aethiopica, as they are the only species with characteristics for phytoremediation that appear in commercial statistics. This allows us to visualize some preference in the use of these plants and the opportunity for their commercialization.

Table 2. Value of production and planted and harvested areas of the main ornamental plants in Mexico [54].

\begin{tabular}{ccccc}
\hline & Planted Surface * (ha) & Harvested Surface * (ha) & Production (Ton) & Production Value (USD) \\
\hline Rosa sp. & 1709.9 & 1635.5 & $13,084,178.4$ & $111,622,031.33$ \\
Chrysanthemum & 2687.2 & 2687.2 & $12,166,948.9$ & $98,736,735.73$ \\
Gladiolus & 4667.8 & 4667.8 & $5,425,054.6$ & $68,772,107.12$ \\
Euphorbia pulcherrima & 275.7 & 275.7 & $19,386,361.6$ & $36,301,162.20$ \\
Dianthus caryophyllus & 640.0 & 549.0 & $4,210,560.0$ & $28,150,581.10$ \\
Gerbera jamesonii & 126.5 & 114.5 & $1,375,830.4$ & $19,449,216.78$ \\
Anthurium andreanum & 1.0 & 1.0 & 1542.7 & $272,865.08$ \\
Zantedeschia aethiopica & 6.5 & 6.5 & 2331.5 & $30,318.34$ \\
\hline
\end{tabular}

* Total data at the national level, do not include crops in constructed wetlands.

Another important strategy to strengthen the use of ornamental plants is production networks. Regarding this, the Macro Ornamental Network [9] that operates in Mexico was established as a strategic plan to join forces and generate knowledge, as well as to preserve and take advantage of native ornamental resources in a sustainable way. The purpose of the network is to promote the sustainable use of plants for the benefit of the society through the participation of educational institutions, research institutions, government agencies, and producer associations. It is observed that the CW phytoremediation species are not constituted as networks, which translates into an area of opportunities for the administration of $\mathrm{CW}$ crops to potentiate the crops.

Among the species analyzed, Zantedeschia aethiopica and Anthurium stand out for their production, [54] and, in the case of heliconia, there is a total of 50 ha cultivated in Mexico [40].

\subsection{Social Potential}

In this study, the social potential was classified as ornamental, artisan, medicinal, and food. Therefore, plant species can be used as raw material in the elaboration of handicrafts, flower arrangements, and the cultivation of seedlings in pots that can be marketed in small quantities, as shown by some works $[13,48]$. At the same time, a culture promoting the efficient use of natural resources would develop in localities where CW planted with ornamental flowers are constructed, such as the Pinoltepec project in the municipality of Emiliano Zapata, Veracruz, Mexico [13]. To take advantage of the social potential that ornamental plants have, it is important to describe their uses considering the benefits they provide to the society, i.e., artisanal, ornamental, cultural, and medicinal benefits (Figure 4). 


\section{Social value of ornamental plants used in constructed wetlands}

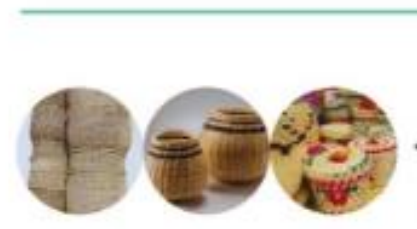

Artisanals

Elaboration of handicrafts such as furniture and baskets, in addition to cellings and handcrafted fences.
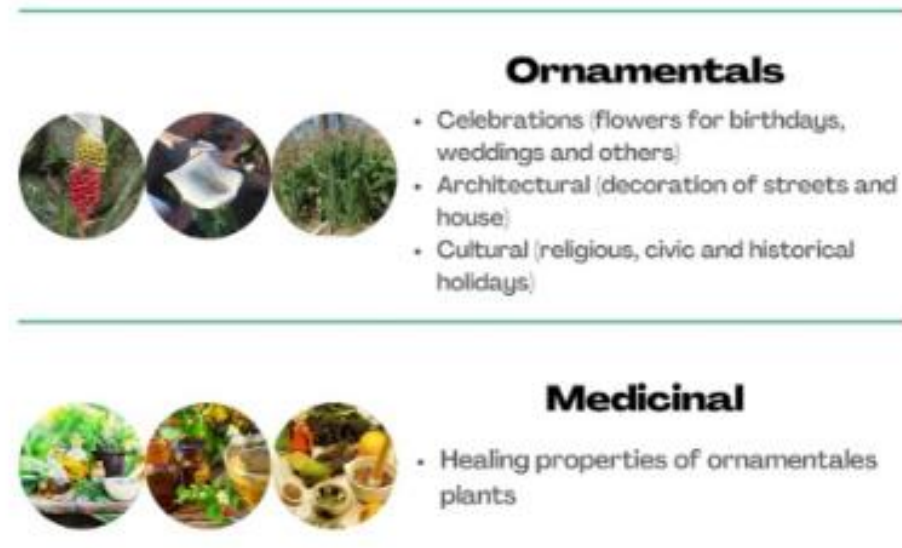

\section{Meclicinal}

Healing properties of ornamentales plants

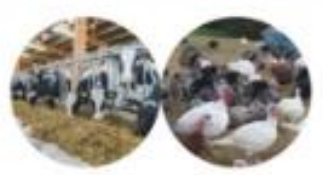

Food

- Stems, leaves, flowers and roots

Figure 4. Classification of the social values of ornamental plants used in constructed wetlands $[9,74]$.

The use of ornamental plants in rural localities, where their cultivation in $\mathrm{CW}$ is feasible, gains importance because these localities regularly lack flower shops to satisfy plant demand for religious events, family, civic, and cultural festivities. Thus, the cultivation of these species may represent an opportunity for local trade: in addition to representing an income-generating opportunity, it may reduce costs due to the expenses of moving to nearby locations for plant acquisition.

Of the phytoremediation plants identified, $90.5 \%$ have an ornamental use, and the rest can be used in artisanal activities; they both have the potential to be commercialized and used in social and cultural local events.

\section{Discussion}

The construction of wastewater treatment systems based on CWs and phytoremediation processes has gained great importance in recent years. Several authors [19,31,47] agree that for pollutants, such as $\mathrm{COD}, \mathrm{BOD}_{5}$, nitrates, phosphates, metals, and some drugs, phytoremediation plants provide removal efficiencies greater than $50 \%$. CWs with phytoremediation plants are an ecotechnology that contributes to the recovery of ecosystems because they supply them with good quality water, in addition to allowing the reuse of second use of water in some non-food activities. Other pollutants are not considered sufficiently in purification studies using ornamental plants, as is the case of ibuprofen (0-50\%), analyzed in CWs vegetated with Spathiphyllum wallisii and As (0-50\%), investigated using species of tame grass and Zantedeschia aethiopica. 
These systems are recommended owing to their low construction, operation, and maintenance costs [11]. Hernández [48] reports that a CW operating in Pinoltepec, Veracruz, with a capacity of $20 \mathrm{~m}^{3}$ / day was built with an investment of 140,000.00 USD, while a conventional system with a capacity of $1080 \mathrm{~m}^{3} /$ day, required an investment of 301,414.86 USD plus the expenses of operation, for a total amount of 1964.63 USD per month.

On the other hand, in an application for the treatment of wastewater of aquatic origin, Betanzo [75] in Veracruz, Mexico, reported an investment of 53,057 USD for the construction of a CW to treat $3108 \mathrm{~m}^{3}$ with a hydraulic retention time of $72 \mathrm{~h}$. In this sense, it is evident that the use of $\mathrm{CW}$ is an economical option in contrast to conventional treatment systems.

One of the main concerns regarding the reproduction of plant species is the spread of invasive species; however, no official records were found classifying some of the 21 ornamental species as invasive. In other countries, the use of Typha latifolia is suggested. However, this plant can invade channels; therefore, its use must be well managed.

As for the economic aspect, the crops that have been used the most in CW projects are Zantedeschia aethiopica and Spathiphyllum blandum. However, Anthurium andreanum has also given good results in phytoremediation [12] and has great commercial value for decoration and as a potted plant [51,58], in addition to its long shelf life [59]. According to previous research [12,55], it can be a convenient option, considering the recommendations according to the type of climate. In a study carried out in the areas of Xalapa and Actopan, [36] through surveys, the preference and acceptance of ornamental plants was determined. It was reported that $84 \%$ of the sample indicated that the plants have potential for sale and distribution because they are attractive for decorating houses, parks, gardens, in addition to being showy and having high durability.

The alternative of wastewater sanitation based on constructed wetlands has given good results at the level of experimentation, but there are few cases analyzed in small towns, such as those that operate in the states of Mexico, Quintana Roo, Michoacán, Morelos, Hidalgo, Oaxaca, and Veracruz [16,73]. This means that this is an area offering opportunities for projects involving social participation and social capital, that may raise awareness of the common good of natural resources [76], as well as promote entrepreneurship [77] to guarantee the continuation of the projects.

In specific cases, the environmental, economic, and social potential of the floristic species cultivated in CWs has been proved in some projects, as is the case of the town of Pinoltepec, Veracruz [50,55], where the commercialization of cultivated hydrophytic plants operates under flooding and in the presence of high concentrations of nutrients. These species are: Typha domingensis, Cyperus papyrus, and C. alternifolius, as well as plants that produce flowers, such as Zantedeschia aethiopica, Lilium spp., Anthurium spp., and Hedychium coronarium. This coincides with the recommendations of other authors $[16,31]$ to use phytoremediation, adding the benefit that this practice promotes social organization through groups that can take charge of the administration of wetlands.

At the international level, there are few $\mathrm{CW}$ projects operated by social groups. In India, there is a record of a $\mathrm{CW}$ operated by a group of women from a cooperative society in Mhaswad. The project was developed in order to facilitate rural development and empower women by implementing a CWs [78].

The benefits that the ecotechnology of constructed wetlands cultivated with ornamental plants would generate if they were implemented in a percentage of the 182,675 localities in Mexico with a population of less than 2500 inhabitants have not been measured [79]. This ecotechnology would contribute to the sanitation of the receiving sources of wastewater discharges, and the local trade of floristic products would be promoted.

The analysis we carried out suggests that it is advisable to link the phytoremediation processes to the environmental, economic, and social potentials of plants, which makes them more attractive in the operation because they represent an opportunity to generate income while, at the same time, contributing to environmental, economic and social sustainability. 
However, the public sector, in particular, municipal authorities, have not shown interest in the implementation of CWs, which has resulted in the absence of alternative treatment infrastructures in small towns [80-82], even though CWs represents a tool for sustainable development. In addition, there are public policies that allow access to financial resources for the construction of CWs, considering that the Operating Rules for the Drinking Water, Drainage, and Treatment Program in charge of Comisión Nacional del Agua, allow sanitation actions "Preferably with technologies that do not require electrical energy, chemical reagents, or specialized labor" [9]. Furthermore, the promotion of community participation is mentioned as a necessary element for the population of small towns to appropriate the alternative technologies and contribute to sustainable local development.

At an international level, the use of ornamental plants for phytoremediation processes has been successfully exploited in various countries. Sandoval et al. [31] report activity in countries such as Brazil, China, Chile, Colombia, Costa Rica, Egypt, India, Kenya, Nepal, Portugal, Spain, Sri Lanka, Taiwan, Thailand, Turkey, USA, Vietnam, and United Kingdom, where they have worked with plants such as Cannas, Zantedeschia aethiopica, Alpinia purpurata, Hemerocallis flava, R. carnea, I. pseudacorus, L. salicaria, Hedychium coronarium, Iris pseudacorus, Iris sibirica, Tulbaghia violácea, Ludwigia inucta, Hedychium coronarium, Polianthus tuberosa, Strelitzia reginae, Anthurium andreanum, Hemerocallis, Dumortieri, Gladiolus spp, Spathiphyllum wallisii, Alocasia spp., Agapanthus africanus, Watsonia borbonica, Hymenocallis littoralis, L. bailey, Crinum asiaticum, Spathiphyllum clevelandii, Eleocharis dulcis, Hemerocallis fulva L., Hibiscus moscheutos L., Aeonium purpureum, Crassula ovate, Equisetum hyemale, Nasturtium, Narcissus impatiens, Anigozanthosand, and Heliconias, with removal efficiency results between $20 \%$ and $99 \%$ for different contaminant indicator parameters.

\section{Conclusions}

This study revealed that the use of the 21 species of ornamental plants in constructed wetlands is a sustainable alternative, since, in addition to having phytoremediation characteristics by removing pollutants, they are raw material for artisanal elaboration, economically favoring their users. In addition to the social aspect, some of these plants have medicinal properties or can simply be used as ornamental plants at home.

Zantedeshia aethipica and Anturium spp. have a high market value (6 million of Mexican pesos in 2018), better vase life, and good aesthetic appearance, up to 25 days in the case of Heliconias, Zingiber spectabile and Anthurium andreanum.

Thus, their use in constructed wetlands is recommended. Training of community groups in the maintenance of wetlands, the use of ornamental vegetation, and the good management of natural resources is also suggested.

Social participation will be essential in the operation and maintenance stage of CWs to guarantee the continuity of projects and good environmental practices, for which the management of government support or derived initiatives is recommended.

Author Contributions: J.L.M.-M. and I.Z.-C.; methodology, M.G.H.y.O.; software, E.A.B.-T.; validation, graphs and figures, M.V.-Á., and L.C.S.-H.; formal analysis, I.Z.-C. and S.A.Z.-C.; investigation, L.C.S.-H., S.A.Z.-C., M.G.H.y.O., E.A.B.-T.; resources, J.L.M.-M.; data curation, J.L.M.-M. and I.Z.-C.; writing—original draft preparation, L.C.S.-H., E.A.B.-T.; writing—review and editing, I.Z.-C. and S.A.Z.-C.; visualization, L.C.S.-H. and E.A.B.-T.; supervision, J.L.M.-M. and I.Z.-C.; project administration, J.L.M.-M.; funding acquisition, J.L.M.-M. and I.Z.-C. All authors have read and agreed to the published version of the manuscript.

Funding: The study Environmental, Economic, and Social Potentialities of Ornamental Vegetation Cultivated in Constructed Wetlands of Mexico" PhD thesis in Regional Sustainable Development. Xalapa, Veracruz, Mexico: El Colegio de Veracruz." received external funding from the Mexican Consejo Nacional de Ciencia y Tecnología, Programa Nacional de Posgrados de Calidad (CONACYT, National Council for Science and Technology). PhD Jose Luis Marín Muñíz for the direction of the $\mathrm{PhD}$ thesis and guides in the development of this article.

Institutional Review Board Statement: Not applicable. 
Informed Consent Statement: Not applicable.

Data Availability Statement: Not applicable.

Conflicts of Interest: The authors declare no conflict of interest.

\section{References}

1. Alimonda, H. Ecologia política latino-americana e pensamento crítico: As vanguardas enraizadas. Desenvolv. Meio Ambiente 2015, 35, 161-168. [CrossRef]

2. ONU (Organización de las Naciones Unidas). Resolución A/RES/70/1 Transformar Nuestro Mundo: La Agenda 2030 Para el Desarrollo Sostenible; Naciones Unidas: New York, NY, USA, 2015; pp. 16-31.

3. Brundtland, G.H. Informe Brundtland. Comisión Mundial para el Medio Ambiente y el Desarrollo de la ONU; Naciones Unidas: New York, NY, USA, 1987; pp. 16-23.

4. Sánchez, R.J. La Bonanza de los Recursos Naturales Para el Desarrollo: Dilemas de Gobernanza; Comisión Económica para América Latina y el Caribe: Santiago, Chile, 2019; pp. 59-91.

5. Foladori, G. Paradojas de la sustentabilidad: Ecológica versus social. Trayectorias 2007, 9, 20-30.

6. CONAGUA (Comisión Nacional del Agua). Numeragua; Gobierno de Mexico: Mexico City, Mexico, 2018 ; pp. $28-34$.

7. Toledo, V.M. La diversidad biológica de México. Nuevos retos para la investigación de los noventa. Ciencias 1994, $34,43-57$.

8. Rzedowski, J. Diversidad y orígenes de la flora fanerogámica de México. Acta Botánica Mex. 1991, 14, 3-21. [CrossRef]

9. Gámez, M.O.; Villavicencio, G.E.; Serrato, C.M.A.; Mejía, M.J.M.; Treviño, C.G.; Martínez, G.L.; Rodríguez, O.M.; Granada, C.L.; Flores, C.M.; Reyes, S.J.; et al. Conservación y Aprovechamiento Sostenible de Species Ornamentals Nativas de México; Servicio Nacional de Inspección y Certificación de Semillas y Universidad Autónoma Chapingo: México City, México, 2016.

10. Singh, O.V.; Jain, R.K. Phytoremediation of toxic aromatic pollutants from soil. Appl. Microbiol. Biotechnol. 2003, 63, 128-135. [CrossRef]

11. Delgadillo-López, A.E.; González-Ramírez, C.A.; Prieto-García, F.; Villagómez-Ibarra, J.R.; Acevedo-Sandoval, O. Fitorremediación: Una alternativa para eliminar la contaminación. Trop. Subtrop. Agroecosyst. 2011, 14, 597-612.

12. Zurita, F.; de Anda, J.; Belmont, M.A. Performance of laboratory-scale wetlands planted with tropical ornamental plants to treat domestic wastewater. Water Qual. Res. J. 2006, 41, 410-417. [CrossRef]

13. Pedraza, L.A.M. Análisis de la Dinámica Género-Gobernanza en Instituciones Comunitarias de Manejo Común de Recursos. Estudio de caso en Pinoltepec. Ph.D. Thesis, El Colegio de Veracruz, Veracruz, México, 2017.

14. Rivas, A. Constructed Wetland at Cucurucho, Municipality of Tzintzuntzan, Michoacán, Mexico. Available online: http:/ / ponce. sdsu.edu/cucuchucho_constructed_wetland.html (accessed on 24 February 2021).

15. Luna-Pabello, V.M.; Aburto-Castañeda, S. Sistema de humedales artificiales para el control de la eutroficación del lago del bosque de San Juan de Aragón. Rev. Espec. Cienc. Químico Biol. 2014, 17, 32-55. [CrossRef]

16. Marín-Muñiz, J.L. Remoción de contaminantes de aguas residuales por medio de humedales artificiales establecidos en el municipio de Actopan, Veracruz, México. Rev. Mex. Ing. Química 2016, 15, 553-563. [CrossRef]

17. Marín-Muñiz, J.L. Humedales construidos en México para el tratamiento de aguas residuales, producción de plantas ornamental y reúso del agua. Agroproductividad 2017, 10, 90-95.

18. Mitsch, W.J.; Gosselink, J. Wetlands; John Wiley and Sons Inc.: New York, NY, USA, 2015.

19. Marín-Muñiz, J.L.; García-González, M.C.; Ruelas-Monjardín, L.C.; Moreno-Casasola, P. Influence of different porous media and ornamental vegetation on wastewater pollutant removal in vertical subsurface flow wetland microcosms. Environ. Eng. Sci. 2018, 35, 88-94. [CrossRef]

20. Sandoval-Herazo, L.C.; Alvarado-Lassman, A.; Marín-Muñiz, J.L.; Méndez-Contreras, J.M.; Zamora-Castro, S.A. Effects of the Use of Ornamental Plants and Different Substrates in the Removal of Wastewater Pollutants through Microcosms of Constructed Wetlands. Sustainability 2018, 10, 1594. [CrossRef]

21. Arias, M.S.A.; Betancur, T.M.B.; Gómez, R.G.; Salazar, G.J.P.; Hernández, A.M.L. Fitorremediación con humedales artificiales para el tratamiento de aguas residuales porcinas. Inf. Técnico 2010, 74, 12-22.

22. Zurita, F.; Castellanos-Hernández, O.A.; Rodríguez-Sahagún, A. El tratamiento de las aguas residuales municipales en las comunidades rurales de México. Rev. Mex. Cienc. Agrícolas 2011, 1, 139-150.

23. Garzón, Z.M.A.; González, Z.J.; García, B.R. Evaluación de un sistema de tratamiento doméstico para reúso de agua residual. Rev. Int. Contam. Ambient. 2016, 32, 199-211. [CrossRef]

24. Machado, A.I.; Beretta, M.; Fragoso, R.; Duarte, E. Overview of the state of the art of constructed wetlands for decentralized wastewater management in Brazil. J. Environ. Manag. 2017, 187, 560-570. [CrossRef] [PubMed]

25. Colicchia, C.; Strozzi, F. Supply chain risk management: A new methodology for a systematic literature review. Supply Chain Manag. Int. J. 2012, 17, 403-418. [CrossRef]

26. Piñar, M.D.L.Á. Gobernanza Ambiental en Destinos Turísticos de Áreas Naturales Protegidas; El Colegio de Veracruz; IETEC, Arana; Veracruz, México, 2012; pp. 79-93.

27. Shaltout, K.H.; Al-Sodany, M.Y.; Eid, M.E. Biology of Common Reed Phragmites Australis (cav.) trin. ex steud: Review And Inquiry; Assiut University Center For Environmental Studies: Assiut, Egypt, 2006; pp. 2-37. 
28. Castañeda, V.A.A.; Flores, L.H.E. Tratamiento de aguas residuales domésticas mediante plantas macrófitas típicas en Los Altos de Jalisco, México. Paakat Rev. Tecnol. Y Soc. 2013, 5, 33.

29. Manuja, S.; Ram, R.; Singh, R.D.; Mukherjee, D. Evaluation of different herbicides for protection of gladiolus (Gladiolus spp.) crop from weeds. Crop Prot. 2005, 24, 921-926. [CrossRef]

30. Ramos-García, M.; Ortega-Centeno, S.; Hernández-Lauzardo, A.N.; Alia-Tejacal, I.; Bosquez-Molina, E.; Bautista-Baños, S. Response of gladiolus (Gladiolus spp) plants after exposure corms to chitosan and hot water treatments. Sci. Hortic. 2009, 121, 480-484. [CrossRef]

31. Sandoval, H.L.C.; Zamora-Castro, S.A.; Vidal-Álvarez, M.; Marín-Muñiz, J.L. Role of Wetland Plants and Use of Ornamental Flowering Plants in Constructed Wetlands for Wastewater Treatment. Rev. Appl. Sci. 2019, 9, 685. [CrossRef]

32. Sandoval-Herazo, M.; Nani, G.; Sandoval, L.; Rivera, S.; Fernández-Lambert, G.; Alvarado-Lassman, A. Evaluación del desempeño de humedales construidos verticales parcialmente saturados para el tratamiento de aguas residuales porcinas. Trop. Subtrop. Agroecosyst. 2020, 23, 38.

33. Zurita, F.; White, J.R. Comparative study of three two-stage hybrid ecological wastewater treatment systems for producing high nutrient, reclaimed water for irrigation reuse in developing countries. Water 2014, 6, 213-228. [CrossRef]

34. Al-Snafi, A.E. Componentes bioactivos y efectos farmacológicos de Canna indica-An Overview. Rev. Int. Farmacol. Y Toxicol. 2015, $5,71-75$.

35. Amaya, T.S.I. Remoción de Cloruros y Fluoruros de Aguas Residuales en Humedales Construidos Sembrados con Mono y Policultivos Ornamentales; Universidad Veracruzana: Veracruz, México, 2016.

36. Gallegos, P.M.P. Remoción de Nutrientes de Aguas Residuales en Humedales a Escala Unifamiliar Sembrados con mono y Policultivo de Plantas Ornamentales. Bachelor's Thesis, Universidad Veracruzana, Veracruz, México, 2016.

37. Martínez, S.J.A. Evaluación de un Humedal Artificial Sembrado con Plantas Ornamentales Para el Tratamiento Aguas Residuales Domésticas Grises. Bachelor's Thesis, Universidad Veracruzana, Veracruz, México, 2018.

38. CONABIO (Comisión Nacional de la Biodiversidad). Cannaceae Canna Indica L. Ciudad de México. Available online: http: / / www.conabio.gob.mx/malezasdemexico/cannaceae/canna-indica/fichas/ficha.htm (accessed on 22 January 2020).

39. Tejeda, A.; Torres-Bojorges, Á.X.; Zurita, F. Carbamazepine removal in three pilot-scale hybrid wetlands planted with ornamental species. Ecol. Eng. 2017, 98, 410-417. [CrossRef]

40. Jerez, E. El cultivo de las heliconias. Cultiv. Trop. 2007, 28, 29-35.

41. Sosa, R.F.M. Cultivo del género Heliconia. Cultiv. Trop. 2013, 34, 24-32.

42. Carrera, L.R. Evaluación de Crecimiento y Viabilidad de Comercialización de Plantas Ornamentales Sembradas en Microcosmos de Humedales, con Diferentes Sustratos y en Sitios con Diferente Temperatura. Master's Thesis, El Colegio de Veracruz, Veracruz, México, 2016.

43. Belmont, M.A.; Cantellano, E.; Thompson, S.; Williamson, M.; Sánchez, A.; Metcalfe, C.D. Treatment of domestic wastewater in a pilot-scale natural treatment system in central Mexico. Ecol. Eng. 2004, 23, 299-311. [CrossRef]

44. Belmont, M.A.; Metcalfe, D.C. Feasibility of using ornamental plants (Zantedeschia aethiopica) in subsurface flow treatment wetlands to remove nitrogen, chemical oxygen demand and nonylphenol ethoxylate surfactants-A laboratory-scale study. Ecol. Eng. 2003, 21, 233-247. [CrossRef]

45. Ramírez-Carrillo, H.F.; Luna-Pabello, V.M.; Arredondo-Figueroa, J.L. Evaluación de un humedal artificial de flujo vertical intermitente, para obtener agua de buena calidad para la acuicultura. Rev. Mex. Ing. Química 2009, 8, 93-99.

46. Zurita, F.; Del Toro-Sánchez, C.L.; Gutiérrez-Lomelí, M.; Rodríguez-Sahagún, A.; Castellanos-Hernández, O.A.; Ramírez-Martínez, G.; White, J.R. Preliminary study on the potential of arsenic removal by subsurface flow constructed mesocosms. Ecol. Eng. 2012, 47, 101-104. [CrossRef]

47. Zurita, F.; Carreón-Álvarez, A. Performance of three pilot-scale hybrid constructed wetlands for total coliforms and Escherichia coli removal from primary effluent-a 2-year study in a subtropical climate. J. Water Health 2015, 13, 446-458. [CrossRef] [PubMed]

48. Hernández, M.E. Humedales ornamentales con participación comunitaria para el saneamiento de aguas municipales en México. Rev. Int. Desarro. Reg. Sustentable 2016, 1, 1-12. [CrossRef]

49. Dennis, D.J.; Doreen, J.; Ohteki, T. Effect of a gibberellic acid 'quick-dip'and storage on the yield and quality of blooms from hybrid Zantedeschia tubers. Sci. Hortic. 1994, 57, 133-142. [CrossRef]

50. Pedraza, L.A.M. Organización Comunitaria Para el Manejo Común: El caso del Humedal Artificial de Pinoltepec, Municipio de Emiliano Zapata. Master's Thesis, El Colegio de Veracruz, Veracruz, México, 2015.

51. Pato, F.A.; Condés, R.L.F.; Vicente, C.F.E. Introducción al Cultivo del Anthurium para Florcortadaen la Región de Murcia; Comunidad Autónoma de la Región de Murcia: Murcia, España, 2007.

52. Zurita, F.; de Anda, J.; Belmont, M.A. Treatment of domestic wastewater and production of commercial flowers in vertical and horizontal subsurface-flow constructed wetlands. Ecol. Eng. 2009, 35, 861-869. [CrossRef]

53. Merino-Solís, M.L.; Villegas, E.; De Anda, J.; López-López, A. The Effect of the Hydraulic Retention Time on the Performance of an Ecological Wastewater Treatment System: An Anaerobic Filter with a Constructed Wetland. Water 2015, 7, 1149-1163. [CrossRef]

54. Sistema de Información Agroalimentaria de Consulta (SIACON-NG). Available online: https://www.gob.mx/siap/documentos/ siacon-ng-161430. (accessed on 21 January 2020). 
55. Sánchez, O.E. Evaluación del Potencial Redox en Humedales Construidos Tratando Aguas Residuales para la Estimación de Producción de Energía Eléctrica. Master's Thesis, Universidad Veracruzana, Veracruz, México, 2016.

56. González, R.D. Remoción de Contaminantes en Aguas Residuales Mediante Humedales Bioingenieriles Domiciliarios en Diferente Tipo de Sustrato y Sembrados con Strelitzia Reginae. Bachelor's Thesis, Universidad Veracruzana, Xalapa, Veracruz, México, 2017.

57. Ramírez-Guerrero, L.; García-Villanueva, E.; Navarrete-Valencia, A.L.; García-Osorio, C.; Arévalo-Galarza, L. Ave del paraíso (Strelitzia Reginae Ait.) aspectos fundamentales para su producción comercial. Agroproductividad 2017, 10, 43-49.

58. Pato, F.A.; Condés, R.L.F.; Vicente, C.F.E. Ensayo de Zantedeschia Híbrida en Perlita; Comunidad Autónoma de la Región de Murcia: Murcia, España, 2007.

59. Gayosso-Rodríguez, S.; Tetumo-García, J.; Hernández-Hernández, L.U.; Estrada Botello, M.A. El Cultivo del Anturio (Anthurium Andreanum); Universidad Juárez Autónoma de Tabasco: Villahermosa, México, 2013; pp. 13-31.

60. Méndez-Mendoza, A.S.; Bello-Mendoza, R.; Herrera-López, D.; Mejía-González, G.; Calixto-Romo, A. Performance of constructed wetlands with ornamental plants in the treatment of domestic wastewater under the tropical climate of South Mexico. Water Pract. Technol. 2015, 10, 110-123. [CrossRef]

61. Pereira, R.L.S.W. Cultivo de Espatifilo (Spathiphyllumsp); EDEBE: Brasilia, Brasil, 2015; pp. 3-10.

62. Martínez, C.M.I. Evaluación de Remoción de Contaminantes de Aguas Grises Mediante Humedales Artificiales con Diferente Tipo de Sustrato y Sembrados con Plantas Ornamentales. Bachelor's Thesis, Universidad Veracruzana, Xalapa, Veracruz, México, 2017.

63. Plantas y flores. Spathiphyllumwallisii. Available online: https://www.plantsrescue.com/spathiphyllum-wallisii/ (accessed on 28 September 2020).

64. Sandoval, H.L.C.; Marín-Muñiz, J.L.; Alvarado, L.A.; Castelán, R.; Ramírez, L.D. Diseño de un Mesocosmo de Humedal Construidos con Materiales Alternativos Para el Tratamiento de Aguas Residuales en la Comunidad de Pastorías Actopan, Ver; Congreso Interdisciplinario de Ingenierías: Veracruz, México, 2016; pp. 35-43.

65. Romero, C.O. The Medicinal Properties of the Alocasia Genus: A Systematic Review. JAASP Res. Pap. 2017, 6, $25-33$.

66. Gardenia Creating Gardens. Hemerodallis (Azucenas). Available online: https://www.gardenia.net/plant-variety/hemerocallisdaylilies (accessed on 15 February 2020).

67. Bela, A.J.; Chifa, C. Posibilidades de uso Medicinal y Alimenticio de Typha Dominguensis Pers. (Typhaceae), Totora; Universidad Nacional de Noreste: Chaco, Argentina, 2000.

68. Grace, J.B.; Harrison, J.S. The biology of canadian weeds.: 73. Typha latifulia L., Typha angustifolia L. and Typha xglauca Godr. Can. J. Plant Sci. 1986, 66, 361-379. [CrossRef]

69. Olguín, E.; Sánchez-Galván, G.; González-Portela, R.; López-Vela, M. Constructed wetland mesocosms for the treatment of diluted sugarcane molasses stillage from etanol production using Pontederia sagittata. Water Res. 2008, 42, 3659-3666. [CrossRef] [PubMed]

70. Programa Master Gardener. Papiro, Papiro Cyperus. Available online: https://wimastergardener.org/article/papyrus-cyperuspapyrus/ (accessed on 5 September 2020).

71. Missouri Botanical Garden. Dieffenbachia Seguine. Available online: http://www.missouribotanicalgarden.org/PlantFinder/ PlantFinderDetails.aspx?kempercode=b589 (accessed on 22 March 2020).

72. Cuevas, C.D. Evaluación de un Humedal Domiciliario Sembrado con Zingiber Spectabile y Strelitzia Reginae en dos Tipos de Sustratos Para el Tratamiento de Aguas Residuales. Bachelor's Thesis, Universidad Veracruzana, Veracruz, México, 2018.

73. Baltazar-Bernal, O.; Zavala-Ruiz, J. Cultivo de Maracas (Zingiber spp.) en la floricultura tropical. Agroproductividad 2012, 5, 20-28.

74. Rendón, C.A.; Fernández, N.R. Plantas con potencial uso ornamental del estado de Morelos México. Polibotánica 2007, 23, 121-165.

75. Betanzo-Torres, E.A. La Acuacultura en México y el uso de Tecnología Biofloc como Alternativa Sustentable: Análisis de Adopción, Desarrollo y Comparativo con otras Tecnologías para el Cultivo de Tilapia (Oreochromis Niloticus). Ph.D. Thesis, El colegio de Vercaruz, Veracruz, Mexico, 2019.

76. Ostrom, E. El Gobierno de los Bienes Comunes. La Evolución de las Instituciones; Fondo del Cultura Económica: Ciudad de México, México, 2000; pp. 25-33.

77. Campaña, L.M.V.; Molina, Q.C.R.; Romero, F.C.A. Las potencialidades artesanales. Un análisis desde la perspectiva de desarrollo sostenible para su revalorización y empresarialidad turística. Retos Turísticos 2015, 14. [CrossRef]

78. Lekshmi, B.; Sharma, S.; Sutar, R.S.; Parikh, Y.J.; Ranade, D.R.; Asolekar, S.R. Circular Economy Approach to Women Empowerment Through Reusing Treated Rural Wastewater Using Constructed Wetlands. In Waste Management as Economic Industry Towards Circular Economy; Springer: Singapore, 2020; pp. 1-10. [CrossRef]

79. Cervera, F.R.; Rangel, G.W. Distribución de la Población por Tamaño de Localidad y su Relación con el Medio Ambiente; Instituto Nacional de Información Estadística y Geográfica: Zapopan, México, 2015; pp. 10-21.

80. CONAGUA (Comisión Nacional del Agua). Inventario Nacional de Plantas Municipales de Potabilización y de Tratamiento de Aguas Residuales en Operación, Diciembre 2018; Conagua: Veracruz, México, 2018; pp. 205-210.

81. Marín-Muñiz, J.L.; Zitácuaro-Contreras, I.; Palma-Cabrera, E. Participación comunitaria para la implementación de humedales para limpiar el agua residual: Caso de estudio en Pastorías, Actopan, Ver. In Miradas Colectivas, Rutas y Aportes de a la Sustentabilidad; Secretaria de Medio Ambiente: México City, México, 2021; pp. 103-118.

82. González, C.E.; Rivas, A. Humedales artificiales para el tratamiento de las aguas residuales generadas en áreas rurales ribereñas al lago de Pátzcuaro. Tláloc 2008, 43, 8-13. 